\title{
Turbo Equalizer with Doppler Compensation for Underwater Channel:
} Experimental Results

\author{
Gun-Woong Park ${ }^{1, a}$, Ji-Won Jung ${ }^{2, b}$ \\ 1,2,Department of Radio Communication Engineering,Korea Maritime and Ocean University, Busan \\ 606-791, Korea \\ agwpark@kmou.ac.kr, bjwjung@kmou.ac.kr \\ Corresponding Author: Ji-Won Jung
}

Keywords: Underwater acoustic communications, Inter symbol interference, Turbo equalization, Doppler estimation, Decision Feedback Equalizer.

\begin{abstract}
In this paper an acoustic underwater communication link is presented. The channel has severe inter symbol interference, we presented an iterative turbo equalization to cope with inter symbol interference induced by reflection of sea-level and sea-bottom for underwater sensor communication channel. Iterative turbo equalizer consists of inner codes and outer codes, we employ decision feedback equalizer as an outer codes and turbo codes as an inner codes. Equalizer and decoder are connected through the interleaving and de-interleaving that update each other's information repeatedly. At the receiver side, we resort to powerful turbo equalization algorithms that iteratively exchange probabilistic information between inner decoder and outer decoder, thereby reducing the error rates significantly. Very important for a successful decoding is the Doppler compensation, which also is discussed. The raw data rate is 1 [Kbps] but after reduction for the error correction coding the net bit rate is $2[\mathrm{kbit} / \mathrm{s}]$, a reliable communication is shown up to the distance of $350[\mathrm{~m}]$.
\end{abstract}

\section{Introduction}

The excessive multipath encountered in underwater sensor communication (USC) channel is creating inter symbol interference (ISI), which is limiting factor to achieve a high data rate and bit error rate (BER) performance. Various different methods to cope with multipath situation have been developed. In addition to ISI, co-channel interference (CoI) is also occurred results from the use of multiple transmitters in UW communication removal of both CoI and ISI is a challenging problem in view of difficult channel conditions. The optimal detector is a maximum likelihood detector (MLD), which can be realized for example by a soft Viterbi algorithm. Due to the length of the impulse response in the UW channel, the number of states in the decoder will be increased. One well proven method to counteract ISI is the decision feedback equalized (DFE), which has been used in many UW communication links [1]. However the use of DFE has difficulties when multipath with a number of arrivals has equal strength or low SNR. The other way to cope with ISI, iterative equalizer is used which constitutes an outer loop in the receiver. An inner loop consists of iterative decoder. The assembly utilizes error correcting power of the iterative codes to get an efficient equalizer [2,3].

In this paper, we study iterative coding based equalization for single carrier USC channel. Among the iterative coding schemes, turbo codes and LDPC codes are dominant channel coding schemes in recent $[4,5]$. This paper decides that turbo coding scheme is optimal for underwater communications system in aspect to performance, packet size, and underwater environments. As an outer code, DFE is used in the paper. As an inner code, the turbo codes are used. This paper also discussed Doppler estimation algorithm by measuring the length of frame.

\section{System Model of Iterative Turbo Equalizer}


Iterative turbo equalizer has better performance than the general equalizer. However, because of using a MAP (Maximum a Posteriori) algorithm, it has the disadvantage of complexity by increased exponentially as the length of the channel impulse response [6]. For this reason, a low-complexity linear equalizer or DFE are used in order to reduce the complexity. In this paper, we consider turbo equalizer with DFE. The baseband model of turbo equalizer is shown in Fig. 1.

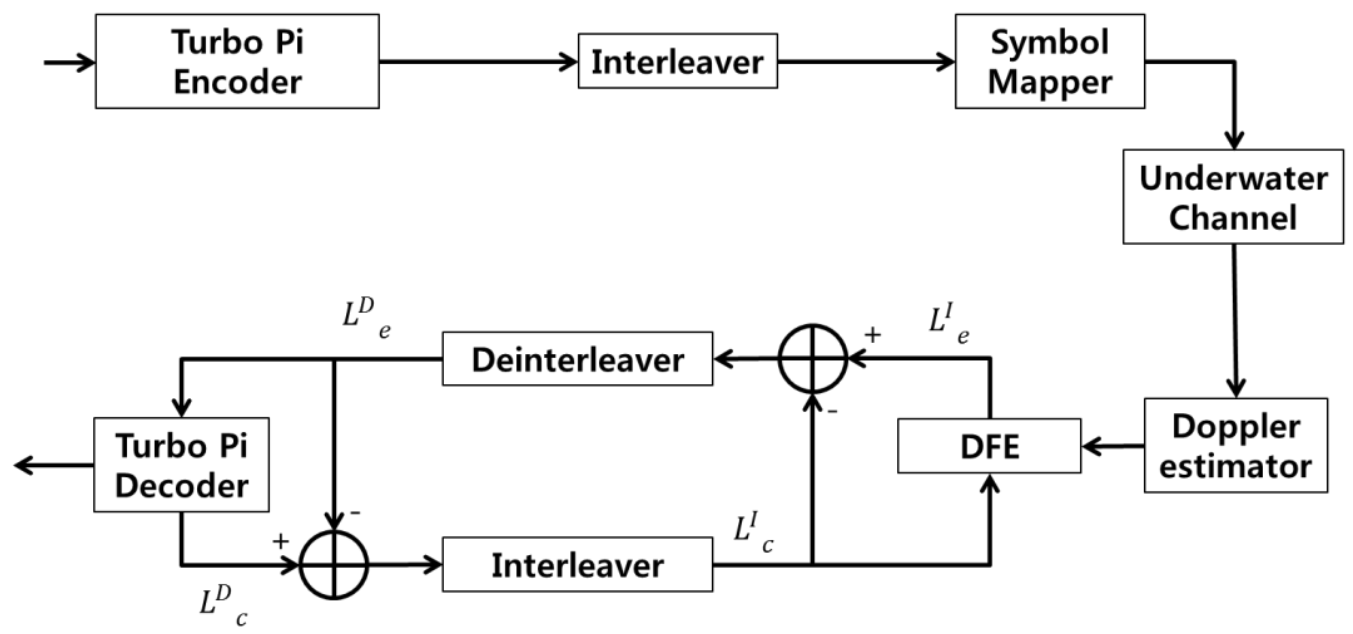

Fig. 1 Model of the turbo equalization in baseband

Fig.1 shows iterative linear equalizer that is decision feedback equalizer is used, which constitutes an outer code of the receiver. An inner code consists of the turbo codes. The information to be transmitted was encoded by a rate of $1 / 2$ turbo code with identical recursive encoders having the duo-binary generator polynomial with 16 states. The interleavers are designed for good properties in a turbo code and were taken from. The receiver of turbo equalizer consists of equalizer and decoder. Equalizer and decoder are connected through the interleaving and de-interleaving that update each other's information repeatedly. The inner coded bits are then subtracted from the input and interleaved. The interleaved output is canceled a posteriori from the proceeding received signal. Interleaving helps receiver convergence.

The $L_{e}^{I}$ is output value of DFE as estimated extrinsic value form received signal. Let $y[k]$ be the equalizer input at time $\mathrm{k}$, then the output of the DFE at time $\mathrm{k}$. $\mathrm{L}_{\mathrm{e}}^{\mathrm{I}}[\mathrm{k}]$ is given by

$$
L_{e}^{I}[k]=\sum_{i=0}^{N_{b}-1} c_{i}[k] y[k-i]-\sum_{j=0}^{N_{a}} b_{j}[k] \widehat{L}_{e}^{I}[k-j]
$$

where, $c_{i}[k]\left(i=0,1, \cdots, N_{c-1}\right)$ are the forward equalizer taps at time $k, b_{j}[k]\left(j=0,1, \cdots, N_{b}\right)$ are the feedback taps at time $\mathrm{k}$, and $\hat{\mathrm{L}}_{\mathrm{e}}^{\mathrm{I}}[\mathrm{k}]$ is the slicer output, which is the constellation point closest to $\mathrm{L}_{\mathrm{e}}^{\mathrm{I}}[\mathrm{k}]$.

and $e_{s}[k]$ is the Sato error given by

$$
\mathrm{e}_{\mathrm{s}}[\mathrm{k}]=\mathrm{L}_{\mathrm{e}}^{\mathrm{I}}[\mathrm{k}] \mathrm{y}[\mathrm{k}]-\gamma \operatorname{sgn}\left\{\mathrm{L}_{\mathrm{e}}^{\mathrm{I}}[\mathrm{k}]\right\} \text {. }
$$

where, $\gamma$ is a constant value and $\operatorname{sgn}\{\cdot\}$ is a sign function defined by

$$
\operatorname{sgn}\{x\}=\left\{\begin{array}{c}
-1 \text { when } x<0 \\
0 \text { when } x=0 \\
+1 \text { when } x>0
\end{array}\right.
$$

The value of $\mathrm{L}_{e}^{\mathrm{D}}$ after interleaving is computed $\mathrm{L}_{\mathrm{e}}^{\mathrm{I}}-\mathrm{L}_{\mathrm{c}}^{\mathrm{I}}$, then input turbo decoder. The estimated extrinsic value of $\mathrm{L}_{c}^{\mathrm{D}}$ at decoder output is given by 


$$
\mathrm{L}_{\mathrm{c}}^{\mathrm{D}}=\log \frac{\mathrm{P}(\mathrm{x}=+1)}{\mathrm{P}(\mathrm{x}=-1)}
$$

The extrinsic value $\mathrm{L}_{\mathrm{c}}^{\mathrm{D}}$ of which calculates the post probability is error correction terms. The reinterleaving of computed value as $\mathrm{L}_{c}^{\mathrm{D}}-\mathrm{L}_{\mathrm{e}}^{\mathrm{D}}$ is input to DFE, then $\mathrm{L}_{\mathrm{c}}^{\mathrm{I}}$ is updated in order to compensate for the errors.

\section{Packet Design and Doppler Estimation Algorithm}

A preamble of known symbols is appended to the beginning of each packet for carrier and clock recovery. Among those methods, data-aided (DA) estimations are normally employed to attain good performance with short preambles [7].

Filtering the received waveform in a matched filter and sampling at proper times yields

$$
x(k)=c_{k} e^{j\left(2 \pi f_{d} k T+\theta\right)}+n(k)
$$

where $c_{k}$ are unit-amplitude symbols, $f_{d}$ is the carrier frequency and $\theta$ is carrier phase. $T$ is symbol duration. $\mathrm{n}(\mathrm{k})$ are complex-valued independent Gaussian random variables. In data-aided mode, modulation can be easily stripped by multiplying $\mathrm{x}(\mathrm{k})$ with $\mathrm{c}_{\mathrm{k}}^{*}$ (complex conjugate of $\mathrm{c}_{\mathrm{k}}$ ) and yields

$$
\begin{aligned}
\mathrm{z}(\mathrm{k}) & =\mathrm{c}_{\mathrm{k}}^{*} \mathrm{c}_{\mathrm{k}} \mathrm{e}^{\mathrm{j}\left(2 \pi \mathrm{f}_{\mathrm{d}} \mathrm{kT}+\theta\right)}+\mathrm{n}(\mathrm{k}) \mathrm{c}_{\mathrm{k}}^{*} \\
& =\mathrm{e}^{\mathrm{j}\left(2 \pi \mathrm{f}_{\mathrm{d}} \mathrm{kT}+\theta\right)}(1+\tilde{\mathrm{n}}(\mathrm{k}))
\end{aligned}
$$

As seen Eq. 6, after demodulation, $\mathrm{z}(\mathrm{k})$ can be viewed as a complex sinusoid embedded in white Gaussian noise. Data-aided carrier frequency and phase estimator is derived based on the observation of a few consecutive samples $\{\mathrm{z}(\mathrm{k}), 0 \leq \mathrm{k} \leq \mathrm{L}-1\}$.

For the purpose of large frequency offset estimation, an improved algorithm is employed.

$$
\hat{\mathrm{f}}_{\mathrm{d}}=\frac{1}{2 \pi \mathrm{T}} \arg \left\{\sum_{\mathrm{m}=1}^{\mathrm{N}-1}\left[\mathrm{R}(\mathrm{m}+1) \mathrm{R}(\mathrm{m})^{*}\right]\right\}
$$

where, $\mathrm{R}(\mathrm{m})$ is the sample correlations.

$$
\mathrm{R}(\mathrm{m})=\frac{1}{\mathrm{~L}-\mathrm{m}} \sum_{\mathrm{k}=\mathrm{m}}^{\mathrm{L}-1} \mathrm{z}(\mathrm{k}) \times \mathrm{z}^{*}(\mathrm{k}-\mathrm{m}) \quad \mathrm{m}=1,2, \cdots, \mathrm{N}
$$

\section{Experimental Results}

The experiment was conducted off the lake of Munkyung city, Korea, during March 2014. For fixed transmission, the transducer was suspended in water at $2[\mathrm{~m}]$ depth and $40[\mathrm{~m}]$ above the bottom, and the source-receiver range was 350[m]. The QPSK signals were employed. The signal structure of the packet is shown in Fig. 2. As can be seen that packet is preceded by a linear frequency modulation (LFM) signals during 0.2 seconds, which we called LFMB (LFM Begin). The LFMB is followed by a gap during 0.5 seconds, plus PN codes of 256 symbols followed by preamble data of 100 symbols. Then, it is the data package consisting of 472 symbols. At the front of the data package, a small block of training data is used for initial channel estimation and Doppler estimation. The whole packet was ended by another LFM signal named as LFME (LFM End), which was separated from the data package with a gap. During transmission, gaps that were sufficiently long were introduced among packets for avoiding inter-packet interference. 




Fig.2 Packet structure

Fig. 3 shows procedure of the demodulation with PN codes and preamble data. The frequency offset resultant from Doppler estimation algorithm described in previous Section is done in the packet of PN codes. After Doppler estimation is finished, phase offset estimation is done in preamble packet. With estimated frequency and phase offset, the whole data packet is compensated. And then the corrected data get into the equalizer.



Fig.3 Frequency and Phase offset compensation

Experiment parameters are listed in Table 1.

Table 1 Parameters of UWA channel Experiment.

\begin{tabular}{cc}
\hline Source & 944 bit text \\
\hline Channel coding & Turbo code \\
Coding rate & $1 / 2$ \\
Input bit size & $944 \mathrm{bits}$ \\
Bit rate & $1[\mathrm{kbps}]$ \\
Center frequency & $16[\mathrm{kHz}]$ \\
Sampling frequency & $192[\mathrm{kHz}]$ \\
Modulation & QPSK \\
Water depth & $50[\mathrm{~m}]$ \\
Distance & $400[\mathrm{~m}]$ \\
Depth & TX : $2[\mathrm{~m}], \mathrm{RX}: 30[\mathrm{~m}]$ \\
\hline
\end{tabular}

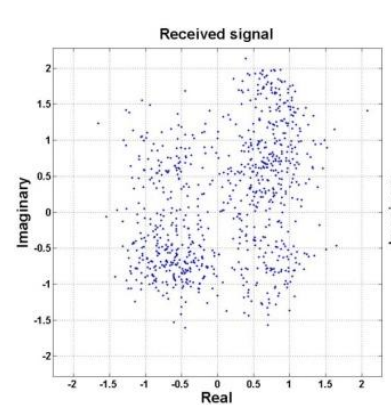

(a)

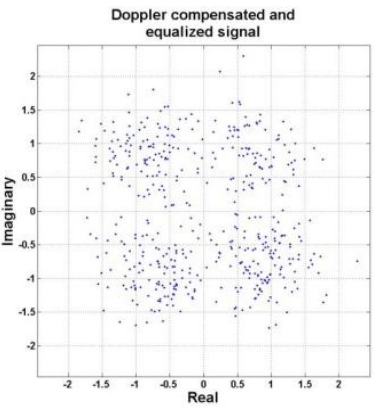

(b)

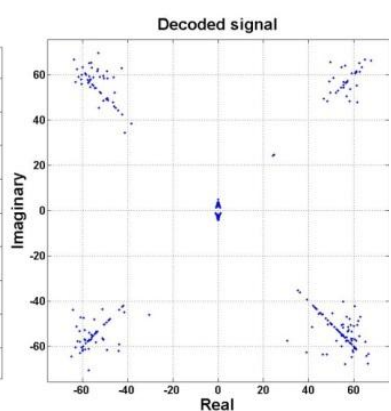

(c)

Fig.4 Scatter plot of (a) received signals (b) Doppler compensated and equalized signals (c) turbo decoded signals 
Fig.4 shows scatter plots received signals, Doppler compensated and equalized signals and turbo decoded signals. As shown in Fig. 4, QPSK constellation is spread by the Doppler and phase distortion (see Fig. 4(a)), After Doppler and phase offset compensation, the signal is constellated in the quadrature points. After turbo decoding, we confirmed that the constellation is very clear Fig.4 clearly show that the receiver model suggested in this paper works well with respect to Doppler estimation and iterative turbo decoding.

Fig. 5 shows the number of errors of proposed system based on block diagram of Fig. 1 and packet structure of Fig.3, Fig. 4. The errors occur 370 bits among 944 coded bits at received signals. After Doppler estimation and equalization, there are 96 bits errors. And then, the iteration is done by turbo equalizer. After first iteration, 85 errors are occurred. All information data are correctly decoded after two iterations. We confirmed that the Doppler estimation algorithm works effectively and the frequency offset is properly compensated and performance gain is obtained by iterations.

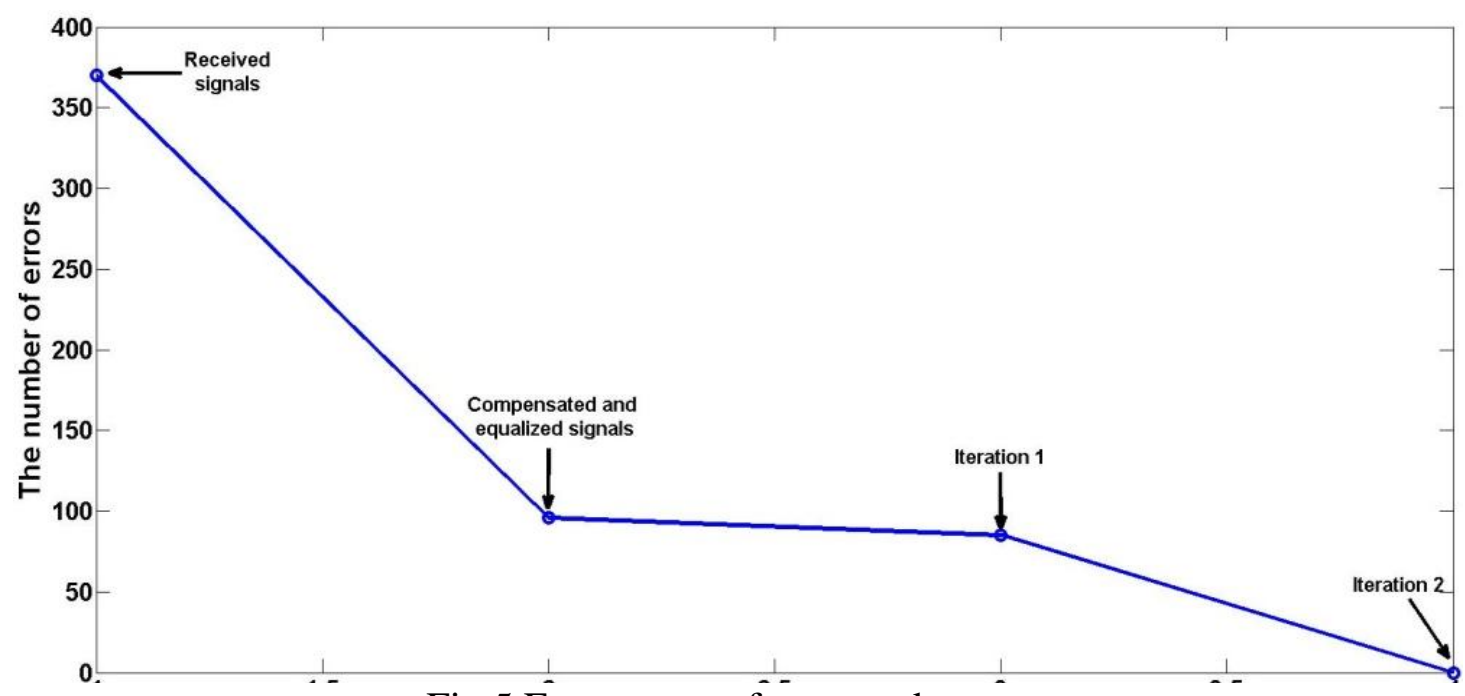

Fig.5 Error curve of proposed system

\section{Conclusions}

In this paper, we proposed receiver structure based on an iterative turbo equalization to cope with inter symbol interference and multipath errors underwater sensor communication channel. Also Doppler estimation algorithm is employed to compensate frequency offset. Iterative turbo equalizer consists of inner codes and outer codes, we employ decision feedback equalizer as an outer codes and turbo codes as an inner codes.

We get the performance of the iterative turbo equalizer with Doppler estimation by experimenting in the Munkyung city, Korea, in March 2014. The distance between transmitter and receiver is $350[\mathrm{~m}]$. The transmitter was deployed in water of $2 \mathrm{~m}$ depth and receiver was fixed in water of $40[\mathrm{~m}]$ depth. In simulation results, distorted received signals were compensated by Doppler estimation and equalization. The number of errors were reduced 370bits to 96bits. After one iteration in turbo equalizer, the performance of proposed algorithm was a little improved. After two iterations, error free was occurred. We confirmed that the performance is a little better as iteration number is increased.

\section{Acknowledgements}

This work was supported by Defense Acquisition Program Administration and Agency for Defense Development under the contract UD130007DD.

\section{References}


[1] M. Stojanovic, J. Catipovic, and J. Proakis: Phase-coherent digital communications for underwater acoustic channels, IEEE Journal of Oceanic Engineering, Jan.(1994), pp. 100-111.

[2] Daniel B. Kilfoyle and Arthur B. Baggeroer: The state of art in underwater acoustic telemetry, IEEE J. Oceanic Eng., Vol.25, no.1, Jan. (2000), pp. 4-27.

[3] R. Koetter, A. C. Singer, and M. Tuchler: Turbo equalization, IEEE Signal Process. Mag., Vol. 21, no. 1, Jan.(2004), pp. 67-80.

[4] C. Douillard, C. Berrou: Turbo Code With Rate- $\mathrm{m} /(\mathrm{m}+1)$ Constituent Convolutional Codes, IEEE Trans.Commun, Vol. 53, no 10, Oct. (2005).

[5] T. Richardson and R. Urbanke: Efficient Encoding of Low-Density Parity Check Codes, IEEE Trans. Information Theory, Vol. 47, Feb. (2001), pp.638-656.

[6] J. Ling, T. Yardibi, X. Su, H. He, and J. Li: Enhanced Channel Estimation and Symbol Detection for High Speed Multi-input Multi-output Underwater Acoustic Communications, J. Acoust. Soc. Am., 125, (2009), pp. 3067-3078.

[7] Shuai Tao, Liang Xu-Wen, Chen Xiao-Ting: Decision-Directed Estimation of Carrier Frequency and Phase for Burst PSK Transmission, IEEE Wireless communications, Sept (2009). 